\title{
Optimising the design stability of cemented paste backfilled stopes
}

\author{
RL Veenstra Newmont Australia, Australia \\ JJ Grobler Newmont Corporation, USA
}

\begin{abstract}
This paper presents an optimisation loop that looked to improve how Newmont's Tanami Operations (NTO) determines its required stable stope strengths. In order to do this, a comparison exercise was completed, in which NTO's current stability assessment method was compared to other popular assessment methods ranging from analytical to numerical methods. From this comparison, it was decided to proceed with a different stability assessment method that uses shear stress reduction. The paper then presents how the use of this method was implemented and presents three case studies on how the new stability assessment method has benefited NTO.
\end{abstract}

Keywords: underground, backfill, stability, modelling

\section{Introduction}

Underground (UG) mines extract mineral resources by excavating voids. The size of these voids is dependent on the type of orebody, the ground conditions, and the resulting mining method. Newmont's Tanami Operations (NTO) currently uses a primary-secondary mining method. In this method the primary voids (referred to as stopes) are excavated first in the sequence with the secondary stopes being excavated later in the sequence. However, in order to maximise the economic recovery of the ore, it is necessary to backfill the primary stopes with a material that is stable when exposed by other primary or secondary stopes. Figure 1 presents a schematic of (a) a single-lift primary-secondary sequence and (b) a double-lift primary-secondary sequence. Note that these sequences are an example and there are many possible excavation sequences.

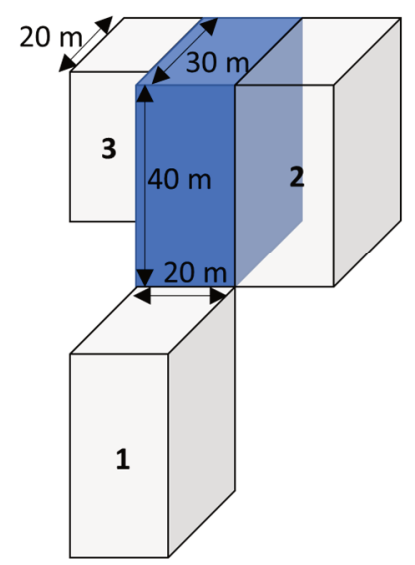

a)

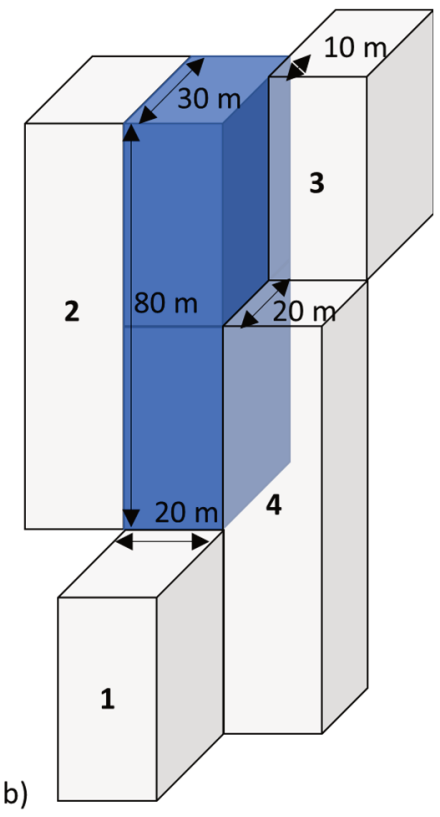

Figure 1 Schematic of (a) Single-lift primary-secondary sequence; (b) Double-lift primary-secondary sequence 
As the mining sequence continues, the stopes in Figure 1 are subjected to a series of vertical or ledge (horizontal and vertical) exposures. The backfill in these stopes needs to be strong enough to remain stable through all these exposures.

Cemented paste backfill (CPB) is an increasingly utilised type of backfill. Its constituent materials are tailings, binder, and water. When initially mixed, it forms a thickened slurry or paste that can be reticulated to the UG stope. However, once in the stope, the binder activates and the CPB gains strength. Therefore, it is necessary to determine the strength required so that the CPB remains stable when exposed.

This paper presents the results of an optimisation exercise where NTO's current design using a semi-analytical method was compared to several common methods for determining the required strength of the CPB. These methods include analytical methods: Mitchell's method (Mitchell et al. 1982); a variation of this method presented by Kuganathan (2005); and a methodology developed at BHP's Olympic Dam (Baldwin \& Grice 1999), as well as numerical modelling using Itasca's FLAC3D (Itasca 2020). It will compare these methods for complexity, ease of use, and potential cost savings due to binder reduction. A case study will then be presented showing NTO's application of the chosen method. Finally, a discussion of future developments will be presented.

\section{Model inputs}

Table 1 summarises the required model inputs for each of the design methods. The analytical methods require the least amount of inputs, while the numerical methods require substantially more inputs. NTO's method is a semi-analytical method that is built from previously completed numerical modelling (Flemmer 2012). The smaller $x$ 's in this method's row are included in the original numerical modelling.

\section{Table 1 Summary of inputs required for stope stability methods}

\begin{tabular}{|c|c|c|c|c|c|c|c|c|c|c|c|c|c|c|}
\hline \multirow[t]{2}{*}{ Method } & \multirow[b]{2}{*}{ 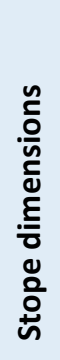 } & \multicolumn{7}{|c|}{ Peak } & \multicolumn{5}{|c|}{ Post peak } & \multirow[t]{2}{*}{ Output } \\
\hline & & 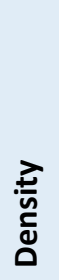 & 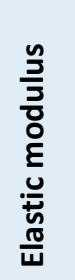 & 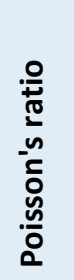 & 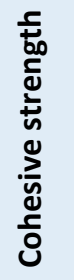 & 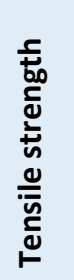 & 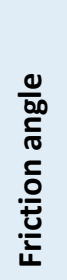 & 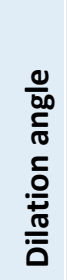 & 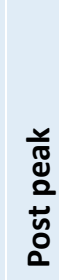 & 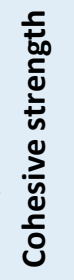 & 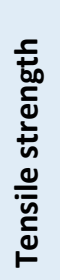 & 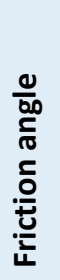 & $\begin{array}{l}\frac{0}{00} \\
\frac{0}{0} \\
\frac{0}{0} \\
.0 \\
\frac{0}{0} \\
\frac{\pi}{0}\end{array}$ & \\
\hline Mitchell's method (Mitchell et al. 1982) & $x$ & $\mathbf{x}$ & & & & & $\mathbf{x}$ & & & & & & & FOS or UCS \\
\hline Olympic Dam (Baldwin \& Grice 1999) & $x$ & $\mathbf{x}$ & & $x$ & & & $\mathbf{x}$ & & & & & & & FOS or UCS \\
\hline Mitchell variation (Kuganathan 2005) & $x$ & $x$ & & & & & & & & & & & & FOS or UCS \\
\hline NTO method (Flemmer 2012) & $x$ & $x$ & $x$ & $x$ & $x$ & $x$ & $x$ & & & & & & & FOS or UCS \\
\hline FLAC3D perfectly plastic & $x$ & $x$ & $x$ & $x$ & $x$ & $x$ & $x$ & $x$ & & & & & & User to define \\
\hline FLAC3D strain softening & $\mathbf{x}$ & $\mathbf{x}$ & $\mathbf{x}$ & $\mathbf{x}$ & $\mathbf{x}$ & $\mathbf{x}$ & $\mathbf{x}$ & $\mathrm{x}$ & $\mathbf{x}$ & $\mathbf{x}$ & $\mathbf{x}$ & $\mathbf{x}$ & $x$ & User to define \\
\hline
\end{tabular}

FOS = Factory of Safety; UCS = unconfined compressive strength

Note that the ultimate outcome of the analytical methods is to determine the backfill mass's required UCS for a given FOS. The UCS test is ubiquitous with backfill laboratory testing (not only for CPB but for essentially all backfills containing binder) as it is a relatively cheap and easy test that can be completed on a mine site. However, it is limited in what other parameters it can provide (though if the testing apparatus is sufficient then the elastic modulus can be estimated). NTO will typically test samples from curing ages of one day to 112 days as part of their standard mix design testing.

Figure 2 presents common models that are used to represent the shear stress versus shear strain behaviour of an older-curing-age CPB UCS sample. The analytical methods presented in this paper all assume rigid 
perfectly plastic behaviour. FLAC3D could be used to represent any of these behaviours, but in this paper, it was used to model elastic perfectly plastic behaviour.

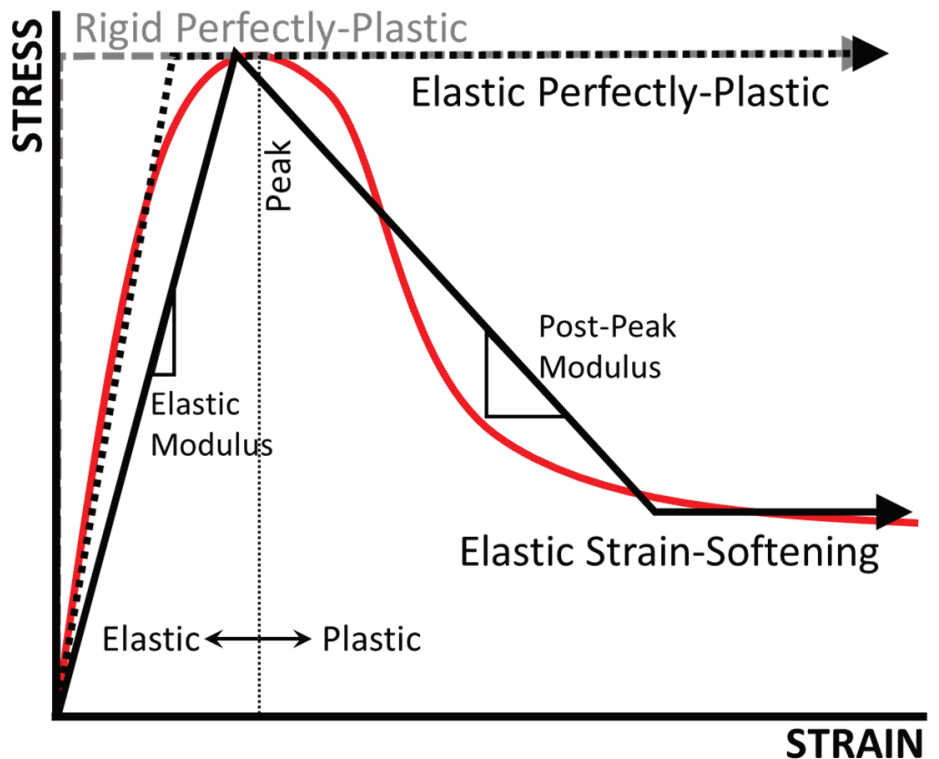

Figure 2 Schematic showing a typical shear stress versus shear strain relationship for CPB (red). Common models for replicating this behaviour are overlayed on the curve: rigid perfectly plastic, elastic perfectly plastic, and elastic strain softening. The elastic and post-peak modulus are shown for the elastic strain softening curve

In order to provide a better understanding of CPB (and to provide more input parameters), NTO has started including consolidated-undrained triaxial testing as part of its standard mix design testing. This allows both the cohesion and friction angle to be determined. These tests were also run into the materials post peak which allowed the residual inputs to be estimated. From these test results a strength model was developed that related the UCS to required model inputs. The strength model was compared to the available quality control data and was found to be similar.

\section{$3 \quad$ Stability methods results}

The stability methods were used to determine the required UCS values in order to obtain an FOS of one for the single-lift and double-lift mining sequences presented in Figure 1. These results would then be used to compare the individual methods. Please note that none of the analytical methods nor NTO's current method could be used to provide a required strength for the first exposure in each sequence (the ledge exposure).

Note that the Olympic Dam, NTO method, and FLAC3D allow for a graduated strength design, meaning that the highest strength material is placed at the bottom of the stope and the strength then decreases with height. This was used in the analysis, but each stope was limited to four individual strength sections.

\subsection{Comparison of stope stability methods}

Figure 3 shows the required UCS versus stope height for the five methods examined. As expected, both graphs generally show the same trends, with the NTO and Olympic Dam methods producing similar results, and that the FLAC3D modelling had the lowest required UCS. Part of the reason for this is that FLAC3D allows the exposures to be modelled more accurately, particularly the 3rd exposure, and the 3rd and 4th exposures in the 40 and $80 \mathrm{~m}$ models, respectively. It also employs better mechanics by using the elastic perfectly plastic model. It was also the only method that was capable of providing an assessment for the ledge exposure. The main detriment to using FLAC3D is that it takes a significantly longer time to produce results and requires considerably more input parameters. Despite this, it was decided to continue using FLAC3D as the stability assessment method at NTO. However, this would require determining a FOS within FLAC3D. 

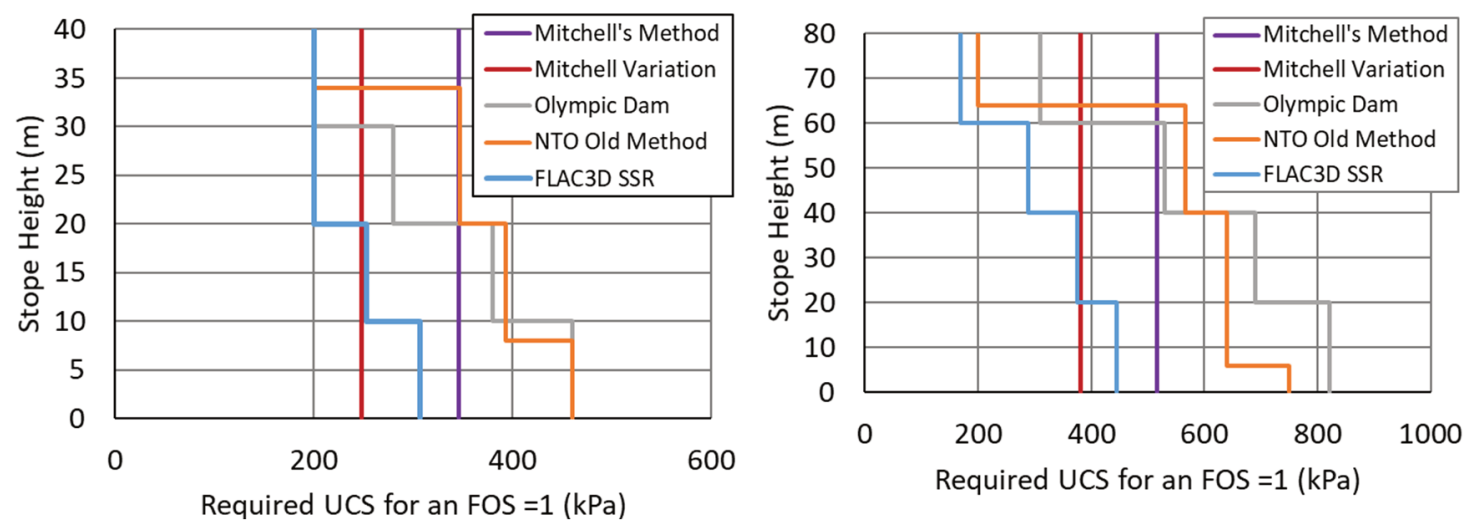

Figure 3 Comparison of the stability methods' required UCS to achieve a FOS of one for the single-lift and double-lift exposure sequence

\subsection{Factor of Safety within FLAC3D}

Generally, the user is required to define what is failure when using FLAC3D. However, recent versions of FLAC3D have included an internal solver that determines the FOS based on the numerical instability of the model. This algorithm has commonly been used in slope stability modelling but has not been employed for determining stope stabilities. In order to determine the FOS, FLAC3D uses a shear strength reduction (SSR) method (Itasca 2020). This involves modifying the strength inputs (in this case the cohesive and tensile strength, and the friction angle or $\phi$ by a safety factor $\left(\mathrm{F}^{\text {Trial }}\right)$ and then running the model. The stability of these trial runs are monitored and are used to bracket the minimum stable safety factor. The strength reduction equations used by FLAC3D are shown below:

$$
\begin{gathered}
\text { Cohesion }^{\text {trial }}=\frac{\text { Cohesion }}{F^{\text {Initial }}} \\
\text { Tension }^{\text {trial }}=\frac{\text { Tension }}{F^{\text {trialial }}} \\
\emptyset^{\text {trial }}=\arctan \left(\frac{\tan \emptyset}{F^{\text {trial }}}\right)
\end{gathered}
$$

Additionally, the authors tried a different method, within FLAC3D, to determine the FOS of a stope. Essentially, all slope stability problems involve the relationship between driving and resisting forces. In the SSR method above, the resisting force is reduced. However, the other method trialled increases the driving force. It does this by incrementally increasing the gravity value by a safety factor within FLAC3D. This is generally referred to as the gravity increase method (GIM). Again, the stability of the model is monitored and the minimum FOS is the last safety factor that remains stable.

\subsection{FLAC3D SSR versus GIM}

A comparison of the two methods was conducted using the single-lift exposure example. The same strength parameters were entered and the FOS of each exposure was obtained using FLAC3D's SSR and the GIM developed by the authors. The results from the three exposures are shown in Figure 4. Each exposure has a velocity and maximum shear strain increment. The velocity plot shows areas where the backfill mass is unstable (red) and stable (blue). The maximum shear strain is useful to show areas in the model where high shear is occurring (red), indicating where the backfill mass is sliding. 


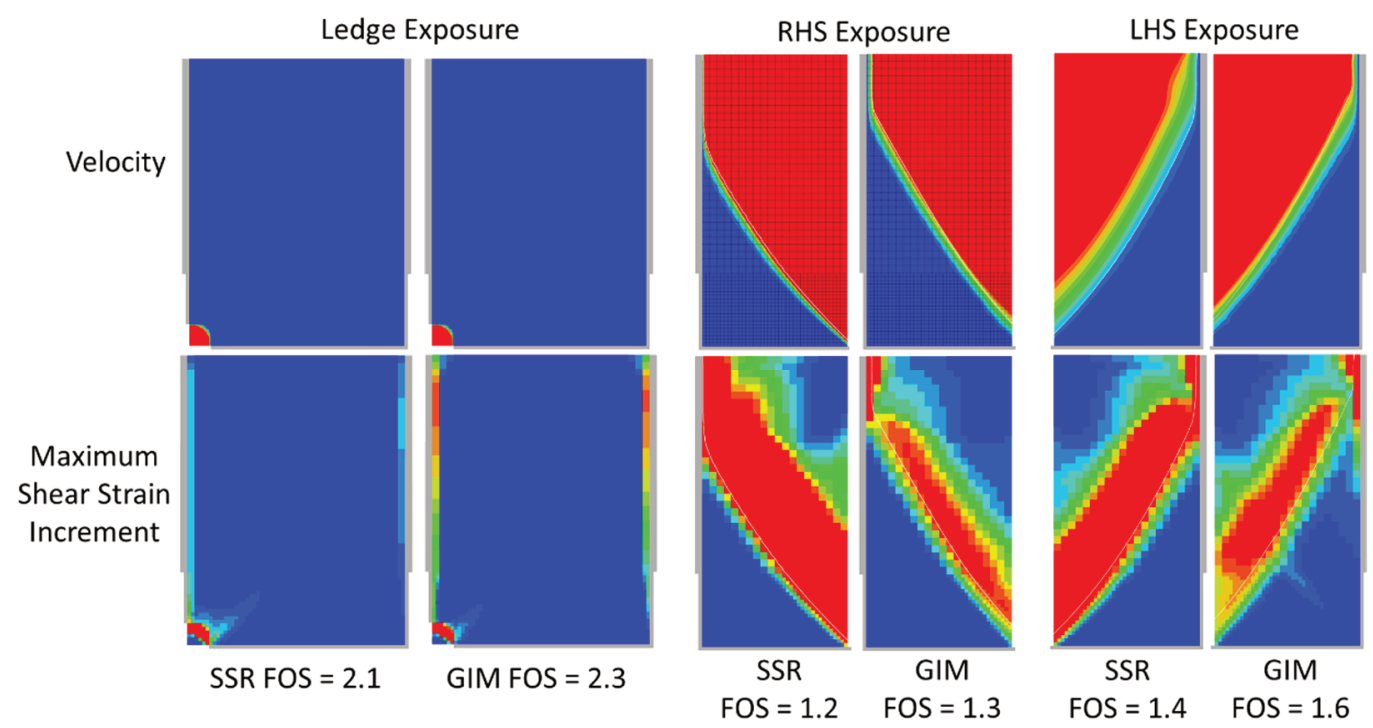

Figure 4 Model plots comparing FLAC3D's SSR against GIM for the three exposures of the $40 \mathrm{~m}$ example

The plots show similar results between the method. However, the SSR method tends to have larger unstable areas, larger shear zones, and a lower FOS. This is due to the slightly different mechanics as the SSR weakens the entire fill while the GIM will concentrate stress on a particular shear zone within the model. However, both methods generate a similar FOS with the GIM's FOS being slightly higher. It was determined to continue using the internal SSR within FLAC3D. However, if the use of elastic strain softening behaviour is required it will be necessary to revisit the use of GIM.

\section{$4 \quad$ Moving forwards with FLAC3D}

After making the decision to move forwards using FLAC3D and its SSR, a calibration exercise was undertaken to determine a minimum design FOS. This involved using NTO as-built design notes to determine what strength of material had been placed at what depths within the stope. Table 2 presents the results of this calibration exercise. As shown in the table, the minimum FOS for any of the modelled exposures was two, which was adapted as the design FOS moving forwards. However, stope performance will be monitored to see if further optimisation is possible.

Table 2 FOS results from investigation of already filled and exposed stopes. Note that non-bolded stopes have not yet to be excavated

\begin{tabular}{|c|c|c|c|c|c|c|c|c|c|c|c|c|}
\hline \multirow{6}{*}{ 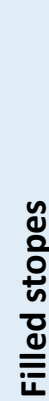 } & \multicolumn{12}{|c|}{ Excavated stopes } \\
\hline & Stope & $058 \mathrm{~F}$ & 052E & $052 F$ & 052G & 055E & 058H & 055F & 055G & O55H & 052J & $055 I$ \\
\hline & 058F & & & & & $>3.0$ & $>3.0$ & 2.5 & & & & \\
\hline & 052E & & & 2.0 & & 2.2 & & & & & & \\
\hline & $052 \mathrm{~F}$ & & & & $>3.0$ & $>3.0$ & & 2.3 & & & & \\
\hline & $052 G$ & & & & & & & & 2.2 & & $>3.0$ & \\
\hline & 055E & & & & & & & 2.1 & & & & \\
\hline & 058H & & & & & & & & & 2.8 & & $>3.0$ \\
\hline
\end{tabular}


Besides the possibilities of analysing the ledge exposures, there are several other advantages to using FLAC3D for determining the required strengths. Some of these advantages include improved mechanics compared to analytical methods (Figure 2), the use of actual stope geometries, the carry-over of damage when looking at multiple exposures, the ability to include time-dependent behaviour, the ability to adapt designs, and the ability to include multiple materials within the stope. The following subsections will explore some of these advantages.

\subsection{Risk mitigation -109 C stope}

109C was a double-lift stope of which the bottom was rockfill and the top portion was filled with CPB. While filling the upper portion of the stope with $\mathrm{CPB}$, the paste plant had an issue with its binder delivery system which overdosed a $5 \mathrm{~m}$ layer of CPB and then underdosed a $10 \mathrm{~m}$ layer of CPB. Specifics of the stope strength zones are shown in part (b) of Figure 5. The underdosed layer reached a UCS of $390 \mathrm{kPa}$ while the underlying overdosed layer reached $1,340 \mathrm{kPa}$. The expected strength was $\sim 1,000 \mathrm{kPa}$ based on the mix design testwork.
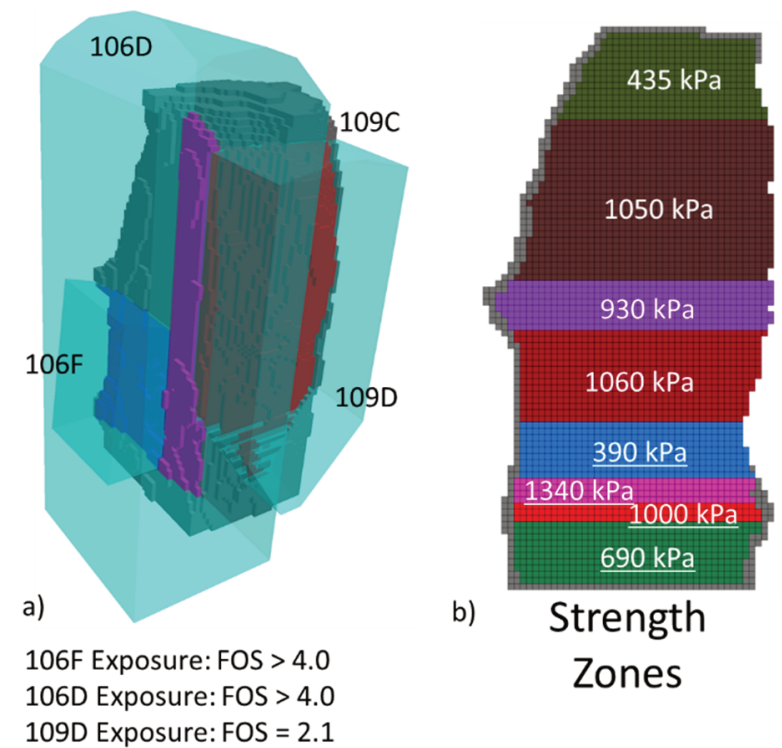

109D Exposure

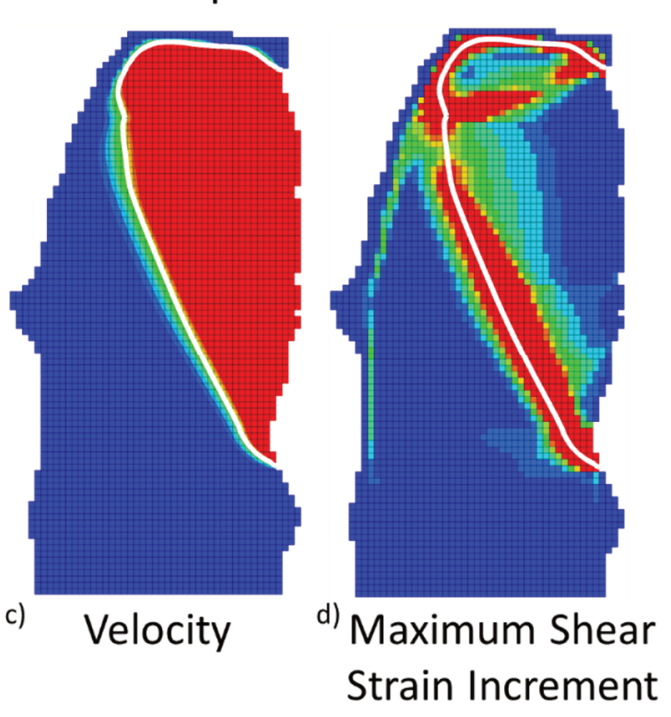

Figure 5 Model plots showing (a) the 109C stope and its exposures, and for the critical 109D exposure the (b) strength zones determined from the stope as-built document; (c) Velocity plots highlighting the stable and unstable zones and (d) high shear areas

The binder addition issues with the plant were identified and fixed but $109 \mathrm{C}$ was left partially empty for a year before filling recommenced. Part of this delay was due to the uncertainty of how the rest of the stope should be filled. The FLAC3D stability method was used to determine the appropriate strengths. This analysis found that a $50 \mathrm{~m}$ layer of $\sim 1,000 \mathrm{kPa}$ material that was overtopped by a weaker $15 \mathrm{~m}$ layer of $400 \mathrm{kPa}$ was sufficient to achieve a FOS of two. Unfortunately, there was another issue with the plant which caused another slightly weaker layer $(930 \mathrm{kPa})$. The result of a design reconciliation determined a FOS of 2.1 (due to both the weak later and the strength overachievement in the other layers) for the critical exposure due to the 109D.

\subsection{Paste-waste project}

To reduce the amount of waste rock being deposited on surface, NTO initiated a project looking at increasing the amount of rock that can be deposited into primary stopes (Veenstra et al. 2020). The basic premise of the project was to encapsulate a cylinder of waste rock within the CPB mass of a primary stope but maintain stope stability. The new stability methodology allowed for an analysis to be completed with a model that contained two very different materials. Figure 6 contains model plots from two different model runs and a cross-section with the cavity monitoring survey surfaces taken during filling. The first model plot shows the 
strength divisions within the model as well as the waste rock cylinder. The second model plot shows the maximum shear strain increment plot, where red indicates the areas where shear is occurring. The first model run was for the original design and the second was a check for the as-built of the stope. This as-built was created from the mine control and paste plant records of backfill deposition, as well as cavity monitoring surveys of the backfill mass that were taken every couple of days. The weak layer in the 085A as-built occurred when the paste plant operator switched to a lower strength zone too early. The use of the new stability method allowed an analysis to be completed of a model with two very different materials. It also allowed for a comparison of the design and as-built models as neither the waste rock or CPB deposition went to plan.
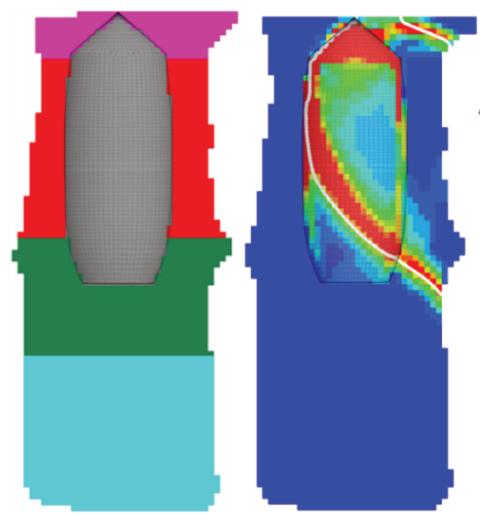

085A Design

FOS $=\mathbf{2 . 0 0}$

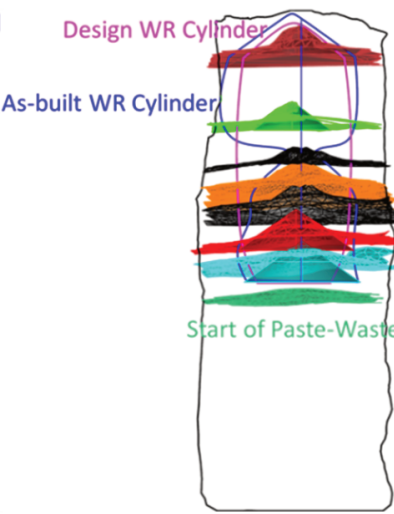

Worst Case As-Built generated from Cavity Monitoring Surveys
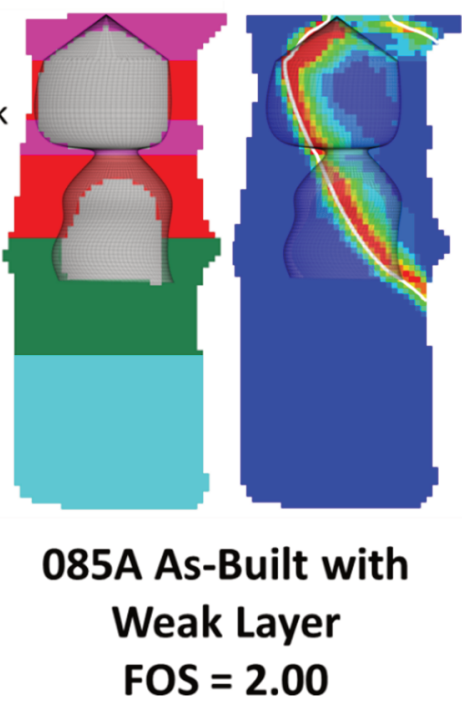

Figure 6 Model plots comparing the 085A design versus as-built. The left plots show the strength divisions, while the right plots show the maximum shear strain increment. The middle plot shows the cavity monitoring surveys undertaken while paste-waste was being deposited

\subsection{Binder cost savings}

The introduction of the new stability method included using the data of a recently completed mix design test programme. A cost analysis was completed comparing the binder costs of the old method and the new stability assessment method. A requirement of this analysis was that the cost savings of this mix design programme be separated from the new analysis method (note that the new method includes the new mix design data). Table 3 summarises the cost savings analysis.

Table 3 Binder cost saving from currently designed stopes. Bolded stopes have been filled and excavated, italicised stopes have been filled but not excavated, and the 145J stope is currently being filled

\begin{tabular}{llll}
\hline Stope & New mix design only & New method & Method change binder cost savings (AUD) \\
\hline 70X & $7 \%$ & $-43 \%$ & $\mathbf{5 8 6 , 6 0 8}$ \\
100D & $10 \%$ & $-13 \%$ & $\mathbf{4 7 4 , 3 2 0}$ \\
O46H & $3 \%$ & $-11 \%$ & 84,700 \\
$091 A$ & $9 \%$ & $-29 \%$ & 527,560 \\
O73G & $-3 \%$ & $-27 \%$ & 215,380 \\
O79D & $7 \%$ & $3 \%$ & $-24,684$ \\
$145 J$ & $4 \%$ & $-14 \%$ & 169,400 \\
& & Total & $\mathbf{2 , 0 3 3 , 2 8 4}$ \\
\hline
\end{tabular}


Seven $100 \%$ CPB stopes have been designed using the new method. The bolded stopes indicate stopes that have been exposed, stopes that are italicised have been filled but not exposed, and the remaining stope (145J) is currently being filled. This shows that the mix design generally increased the binder cost. This was due to a relatively large increase in density but a small decrease in required binder content. However, the overall method change, including the additional mix design costs, was still able to generate cost savings of over AUD 2,000,000. Two of the stopes have been exposed with neither causing more dilution than expected. The other stopes will continue to be monitored.

\section{$5 \quad$ Conclusion and future work}

This paper presents an optimisation exercise looking to improve how NTO determined the required UCS of its stopes. This involved comparing its current method to several popular methods being used to determine the required UCS. Of these methods, FLAC3D was the method chosen, particularly its built-in SSR method. This method reduces the model's strength parameters by a safety factor and monitors each model run for stability in order to determine the minimum FOS. FLAC3D had benefits over the other methods given the type of stope, stope size, and geometry that NTO currently uses as well as utilising a better mechanical model. It also gave the mine flexibility to adapt designs that were not possible with either NTO's original or the other stability methods examined.

In order to determine a design FOS, a calibration exercise of previously filled and exposed stopes was undertaken, which determined a design FOS of two. Using this value, three case studies were presented. The first case study showed the versatility of the new stability method as it allowed for the analysis of a stope with multiple strength zones as well as large differences in filling times. The second case study presented an analysis of a paste-waste. Again, the new stability method allowed for increased flexibility but incorporating two very different materials as well as multiple strength zones. The third and final case study is a cost analysis looking at binder cost savings due to using the new stability method versus NTO's old stability method. The savings so far are around AUD 2,000,000.

These case studies, and the calibration exercise, highlight the importance of creating and updating stope backfill as-builts. These records allow for the easy determination of how a stope was filled and allows for design reconciliation.

In general, NTO has been satisfied with how the new stability method is performing but stope monitoring will continue. Moving forwards, depending on whether the use of elastic strain softening behaviour is required, it may be required to revisit the gravity increase method.

\section{References}

Baldwin, G \& Grice, AG 1999, 'Engineering the new Olympic Dam backfill systems', in G Chitombo (ed.), Proceedings of MassMin2000, The Australasian Institute of Mining and Metallurgy, Melbourne, pp. 351-357.

Flemmer, A 2012, Newmont Tanami Operations Void Fill Note, Newmont Asia Pacific, internal communication.

Itasca Consulting Group Inc. 2020, FLAC3D: Fast Lagranian Analysis of Continua in 3 Dimensions, version 7.0, Itasca Consulting Group Inc., Minneapolis.

Kuganathan, K 2005, 'Geomechanics of mine fill', in Y Potvin, E Thomas \& AB Fourie (eds), Handbook on Mine Fill, Australian Centre for Geomechanics, Perth.

Mitchell, RJ, Olsen, RS \& Smith, JD 1982, 'Model studies of cemented tailing used for mine backfill', Canadian Geotechnical Journal, vol. 19 , no. 1 , pp. 14-28.

Veenstra, RL \& Grobler, JJ 2020, 'Paste-waste design and implementation of Newmont Goldcorp's Tanami Operation', in F Hassani, J Palarski, V Sokoła-Szewioła \& G Strozik (eds), Proceedings of the 13th International Symposium on Mining with Backfill, CRC Press, London, pp. 382-397. 